\title{
Role of RANK and Akt1 activation in human osteosarcoma progression: A clinicopathological study
}

\author{
JIANXI ZHU, YUWEI LIU, YONG ZHU, MIN ZENG, JIE XIE, PENGFEI LEI, KANGHUA LI and YIHE HU \\ Department of Orthopedics, Xiangya Hospital of Central South University, Changsha, Hunan 410008, P.R. China
}

Received August 9, 2015; Accepted January 20, 2017

DOI: $10.3892 /$ etm.2017.4360

\begin{abstract}
The receptor activator of nuclear factor $\kappa \mathrm{B}$ (RANK) axis is the fundamental signaling pathway in bone formation as well as bone tumor pathophysiology. The aim of the present study was to evaluate the impact of the expression of RANK and its downstream signaling molecule Aktl on tumor progression in patients with osteosarcoma. Expression of RANK and Akt1 was examined in 78 human osteosarcoma samples by immunohistochemistry using formalin-fixed samples. Following this, each graded immunohistochemistry result was correlated with clinicopathological parameters and patient survival. In total, 60 osteosarcomas (76.9\%) expressed RANK and 58 cases (74.4\%) showed expression of Akt1. In addition, expression of RANK was negatively correlated with disease-free survival by Kaplan-Meier analysis. A resistance was observed to chemotherapy in RANK-expressing cases, which was statistically significant $(\mathrm{P}<0.05)$. In addition, chemotherapy and staging of the tumor were found to independent factors that have an effect on patient survival $(\mathrm{P}<0.05)$. Thus, RANK was identified as a negative prognostic factor of osteosarcoma survival.
\end{abstract}

\section{Introduction}

Osteosarcoma (OS) is the most prevalent primary bone related malignancy, accounting for $\sim 20 \%$ of primary malignancies of bone among children and adolescents (1). Receptor activator of nuclear factor $\kappa \mathrm{B}$ (RANK) is key to the RANK/receptor activator of nuclear factor $\kappa \mathrm{B}$ ligand (RANKL)/osteoprotegerin $(\mathrm{OPG})$ cascade and regulates a range of physiological

Correspondence to: Dr Kanghua Li or Dr Yihe Hu, Department of Orthopedics, Xiangya Hospital of Central South University, 87 Xiangya Road, Kaifu, Changsha, Hunan 410008, P.R. China E-mail: 199340522@qq.com

E-mail: huyihe1964@163.com

Abbreviations: RANK, receptor activator of nuclear factor $\kappa \mathrm{B}$; RANKL, receptor activator of nuclear factor $\kappa \mathrm{B}$ ligand; OPG, osteoprotegerin; PI3K, phosphatidylinositol 3-kinase

Key words: RANK, Akt1, osteosarcoma, immunohistochemistry processes. Most notably, RANK signaling is essential for bone homeostasis due to its role in the differentiation and activation of bone-absorbing osteoclasts (2). Notably, a previous review has reported that deregulation of the RANK signaling pathway is involved in the progression of multiple human diseases (3). The development of molecular therapies aimed at inhibiting the RANK pathway has been prominent, and a better understanding of this pathway is crucial for future utilization of these evolving drugs. In cancer, the importance of RANK (4) has been highlighted in multiple studies (5-8) that demonstrate its role in the acceleration of cancer metastasis to the bone.

The RANK downstream signal transducer, Akt, also known as protein kinase B (PKB), is essential in the phosphatidylinositol 3-kinase (PI3K) pathway and is important in the maintenance of numerous cellular processes, including cell proliferation, differentiation and metabolism (9). Three distinct genes encode for Akt1 (PKBa), Akt2 (PKBb) and Akt3 (PKBc), and for proteins sharing up to $80 \%$ amino acid sequence identity in mammalian cells (10). Evidence indicates that Akt1 is crucial for the initiation and metastasis of various cancers by agonizing its downstream effectors glycogen synthase kinase-3a/b and mechanistic target of rapamycin, whereas Akt2 antagonizes this effect to inhibit cancer progression (10). However, in lung carcinoma studies a contradictory result is observed (11), which reveal that Akt1 inhibits tumor cell migration and metastasis by promoting relevant protein degradation while Akt 2 does the opposite. In particular, constitutively active protein kinase A signaling due to the loss of the bone tumor suppressor gene resulted in considerable amounts of Akt, which is essential in signal transduction of physiological processes as well as in neoplasia formation in the PI3K/Akt pathway. In addition, part of the tumorigenic effect of RANK may be due to stimulation of the activation of Akt $(12,13)$. A feature of a variety of human cancers, including lung cancer (6), is uncontrolled activation of the Akt pathway.

Therefore, it is hypothesized that RANK participates in the initiation and metastasis of OS partially through the activation of Akt. In the present study, the expression of RANK and Akt1 in 78 OS and 15 osteochondroma patient samples was analyzed by immunohistochemistry in order to characterize the potential interplay between the RANK and Akt factors and their association with tumor progression and metastasis. 
Table I. Clinicopathological data of patients with osteosarcoma and positive RANK or Akt1 expression.

\begin{tabular}{|c|c|c|c|c|c|}
\hline \multirow[b]{2}{*}{ Characteristics } & \multicolumn{3}{|c|}{ RANK positive } & \multicolumn{2}{|c|}{ AKT1 positive } \\
\hline & $\mathrm{N}$ & $\mathrm{N}(\%)$ & P-value & $\mathrm{N}(\%)$ & P-value \\
\hline Gender & 78 & $60(76.9)$ & $>0.05$ & $58(74.4)$ & $>0.05$ \\
\hline Male & 46 & $36(78.2)$ & & 33 (71.7) & \\
\hline Female & 32 & $24(70.5)$ & & $25(78.1)$ & \\
\hline Age & & & $>0.05$ & & $>0.05$ \\
\hline$>21$ years & 42 & $33(78.6)$ & & $30(1.4)$ & \\
\hline$\leq 21$ years & 36 & $27(75.0)$ & & $28(77.8)$ & \\
\hline Histological type & & & $>0.05$ & & $>0.05$ \\
\hline Osteoblastic & 31 & $27(87.1)$ & & $23(74.2)$ & \\
\hline Chondroblastic & 15 & $10(66.6)$ & & $11(73.3)$ & \\
\hline Telangiectatic & 12 & $9(75.0)$ & & $8(66.7)$ & \\
\hline Fibroblastic & 18 & $13(72.2)$ & & $14(77.8)$ & \\
\hline Giant cell & 2 & $1(50.0)$ & & $2(100.0)$ & \\
\hline Staging & & & 0.05 & & $<0.05$ \\
\hline Enneking $1 \& 2 \mathrm{~A}$ & 37 & $24(64.9)$ & & $23(62.2)$ & \\
\hline Enneking 2B\&3 & 41 & $36(87.9)$ & & $35(85.4)$ & \\
\hline Chemotherapy & 50 & $41(82.0)$ & $<0.05$ & $39(78.0)$ & $<0.05$ \\
\hline Responder & 21 & $14(66.7)$ & & $13(61.9)$ & \\
\hline Non-responder & 29 & $27(93.1)$ & & $26(89.7)$ & \\
\hline
\end{tabular}

RANK, receptor activator of nuclear factor $\kappa B$.

\section{Materials and methods}

Patient and sample preparation. In total, 78 patients with primary OS were screened from the archives of the Department of Pathology of Xiangya Hospital of Central South University (Changsha, China). Two independent pathologists in this department reached a consensus to confirm each patient diagnosis. Next, a block of formalin-fixed, paraffin-embedded biopsy material without neoadjuvant chemotherapy and surgery was obtained for staining in the present study. Based on pathological classification, 31 osteoblastic, 18 fibroblastic, 15 chondroblastic, 12 teleangiectatic and 2 giant cell OS samples were selected. Surgery of all patients was undertaken at the Department of Orthopedics of Xiangya Hospital between 2008 and 2014 (Table I). In addition, Enneking staging (14) was utilized as the surgical classification for all cases. As a negative control, 15 (9M/6F) femoral or tibial osteochondroma samples from 2012 to 2014 were collected in the Department of Orthopedics of Xiangya Hospital (Changsha, China). These samples were from 9 males and 6 females, with a mean age of 22.4 (8-37 years). The Medical Review Board of the Central South University approved the study protocol. Furthermore, informed patient consent was obtained prior to the study.

Immunohistochemistry. Consecutive $4-\mu$ m paraffin-embedded sections were stained with antibodies against RANK and Akt1 (ab13918, ab54752, 1:150; Abcam, Cambridge, MA, USA) in accordance with the manufacturer's instructions. In brief, target retrieval was performed by heating in citrate buffer (pH 6.0) at $99^{\circ} \mathrm{C}$ for $30 \mathrm{~min}$. The samples were then incubated with the primary antibody overnight at $4^{\circ} \mathrm{C}$. The following day, Goat anti-mouse secondary antibody (ZDR-5307, 1:100, ZSGB-BIO, Beijing, China) was added and the samples were incubated for $1 \mathrm{~h}$ at room temperature. Images were then captured using a detection system (Leica Microsystems GmbH, Wetzlar, Germany). A negative control was set, by omitting the primary antibody, in order to measure the non-specific conjugation of antibodies. In total, 15 osteochondromas served as the control group. Quantitative assessment was performed under high-power magnification $(\mathrm{x} 400)$ by analyzing the protein expression in 500 adjacent tumor cells. The final score was calculated by combining a score allocated according to the percentage of positive tumor cells ( 0 , none; 1 , $1-29 \% ; 2,30-69 \%$ and $3,70-100 \%)$ and a score for expression intensity $(0$, none; 1 , weak; 2 , moderate and 3 , strong). The result was then divided into negative $(-, 0)$, weakly positive $(+, 1-4)$, median positive $(++, 5-8)$ and strongly positive $(+++$, 9-12) on the basis of the final score value. Finally, the scores were accepted if the two investigators agreed with the values. Otherwise, the values were re-estimated until a consensus was reached. The conclusions were in complete agreement in $80 \%$ of the cases, which indicated that the scoring method was highly reproducible. Thus, all results were evaluated independently by two investigators in order to reach a consensus.

Statistical analysis. All statistical analyses were performed using Statistical Product and Service Solutions version 21.0 software (IBM SPSS, Armonk, NY, USA). An association analysis of RANK and Akt1 with each other and with their respective clinicopathological parameters was performed 

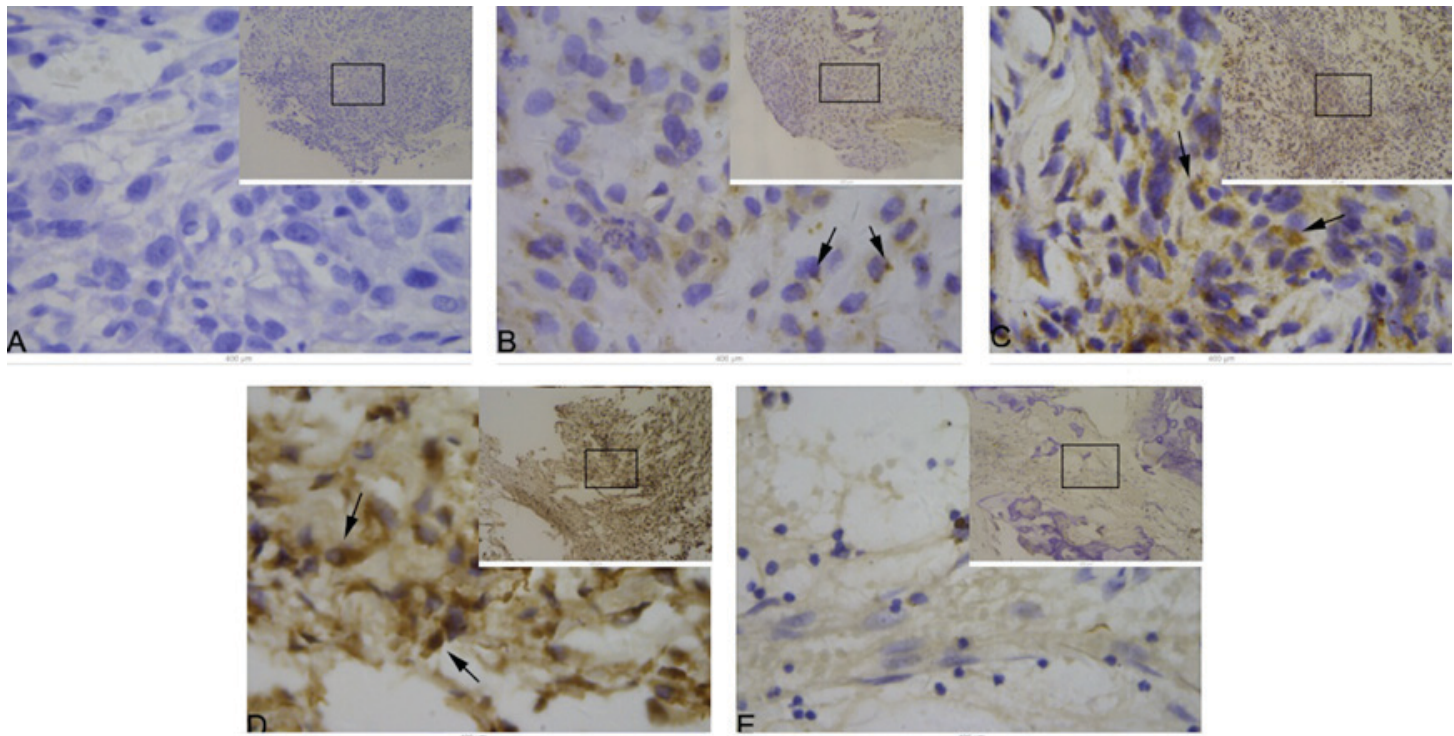

Figure 1. RANK expression in human osteosarcomas and osteochondromas. Representative images are shown for samples that are (A) osteosarcoma negative, (B) osteosarcoma weakly positive, (C) osteosarcoma positive, (D) osteosarcoma strongly positive and (E) osteochondroma negative. Magnification 10x20 for the inset and 10x100 for the main image, which is a magnified image of the area defined by the black rectangle in the inset. RANK, receptor activator of nuclear factor $\mathrm{\kappa B}$.
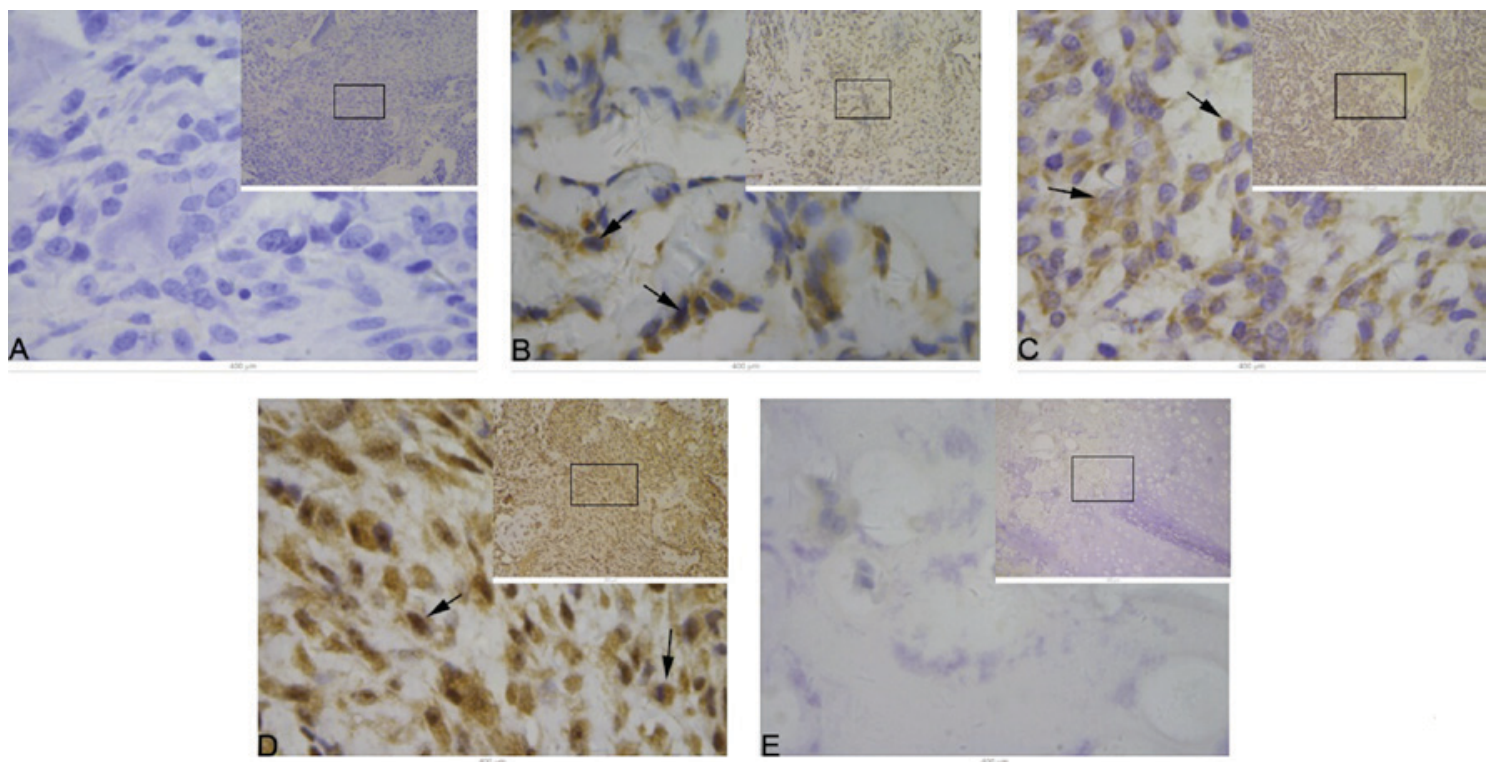

Figure 2. Akt1 expression in human osteosarcomas and osteochondromas. Representative images are shown for (A) osteosarcoma negative, (B) osteosarcoma weakly positive, (C) osteosarcoma positive, (D) osteosarcoma strongly positive and (E) osteochondroma negative. Magnification 10x20 for the inset and $10 \times 100$ for the main image, which is a magnified image of the area defined by the black rectangle in the inset.

using Pearson's $\chi^{2}$ test. In addition, survival analysis was conducted by the Kaplan-Meier method and Cox regression method was utilized in the multivariate analysis. A two-sided $\mathrm{P}<0.05$ was considered to indicate a statistically significant difference.

\section{Results}

Immunohistological detection of RANK and Akt1. The expression of RANK and Akt1 was positive in $60(76.9 \%)$ and 58 (74.4\%) of OS patients, respectively. The specific results are shown in Table I. Of these cases, RANK expression was detected as weak in $28(46.7 \%)$, moderate in $19(31.7 \%)$ and strong in $13(21.7 \%)$ cases, while Akt1 exhibited 30 (51.7\%) weak, $19(32.8 \%)$ moderate and $9(15.5 \%)$ strong expression. Representative images of RANK and Aktl staining in OS and osteochondroma tissues are shown in Figs. 1 and 2. In addition, correlation analysis demonstrated a strong positive association between RANK and Akt1 with a coefficient $\mathrm{r}=0.797(\mathrm{P}<0.001)$ using the Spearman correlation method. The detailed expression results for RANK and Akt1 are shown in Table II.

Clinicopathological correlation of RANK and Akt1 expression. Among the 78 cases of OS, 46 were male and 32 were female 
Table II. Correlation of RANK and Akt1 expression (n).

\begin{tabular}{lrrrr}
\hline & RANK - & RANK + & RANK ++ & RANK +++ \\
\hline AKT1 - & 15 & 3 & 1 & 1 \\
AKT1 + & 2 & 21 & 6 & 1 \\
AKT1 ++ & 1 & 4 & 10 & 4 \\
AKT1 +++ & 0 & 0 & 2 & 7 \\
\hline
\end{tabular}

Coefficient of correlation, $\mathrm{r}=0.797, \mathrm{P}<0.001$. RANK, receptor activator of nuclear factor $\kappa \mathrm{B}$.

Table III. Multivariate survival analysis of patients with osteosarcoma.

\begin{tabular}{lcll}
\hline Factor & Relative risk & $95 \%$ CI & P-value \\
\hline Chemotherapy response & 3.20 & $1.50-6.82$ & 0.003 \\
Enneking staging & 3.01 & $1.40-6.84$ & 0.005 \\
RANK & 8.77 & $1.11-68.969$ & 0.039
\end{tabular}

RANK, receptor activator of nuclear factor $\kappa \mathrm{B}$; CI, confidence interval.

patients. In total, 50 patients received conventional chemotherapy following surgery. The patient follow-up duration ranged between 2 and 44 months (median, 17 months with a standard deviation of 10.8 months), determined from the day of the surgery. In the whole follow-up period, 31 patients (39.7\%) developed lung metastases and 4 patients (5.1\%) had restricted local recurrence. In addition, 28 patients $(23.1 \%)$ succumbed to tumor progression while 11 patients $(14.3 \%)$ succumbed to other causes (Table I). The expression patterns of RANK or Aktl exhibited no statistically significant differences according to patient gender. Furthermore, the expression patterns of RANK or Akt1 demonstrated no statistically significant differences according to the age ( $>21$ or $\leq 21$ years) of the patients.

Patients receiving chemotherapy who showed no signs of metastasis, local recurrence or mortality were classified as responders while the rest were classified as non-responders. A good response to chemotherapy was negatively correlated with the development of metastases $(r=-0.683, P<0.05)$ and also associated with RANK $(\mathrm{P}<0.05)$ and Akt1 $(\mathrm{P}<0.05)$ expression.

Survival analysis for RANK and Aktl. The prognostic significance of RANK and Aktl was examined in OS by reclassification of the cases into lower (comprising negative and weakly positive in previous analyses) and higher (comprising positive and strongly positive in previous analyses).

In total, 38 patients were recruited for the survival analysis. Kaplan-Meier analysis displayed a significantly differentiated expression pattern between lower $(-,+)$ and higher $(++,+++)$ expression and the survival rate was lower in patients with RANK-expressing tumors (Fig. 3).In addition, Akt1-expressing cases demonstrated a similar pattern with regard to the survival

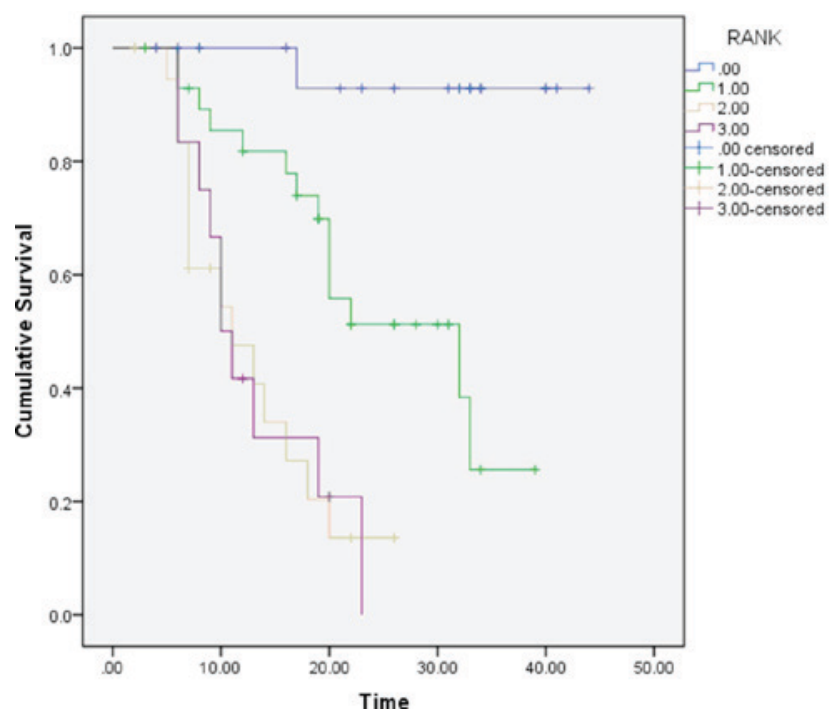

Figure 3. Survival analysis of RANK. RANK, receptor activator of nuclear factor $\kappa$ B. blue, 0.00: RANK- (negative), green, 1.00: RANK+ (weakly positive), yellow, 2.00: RANK++ (median positive) and purple, 3.00: RANK+++ (strongly positive). $\mathrm{X}$ axis, months; $\mathrm{Y}$ axis, survival rate (\%).

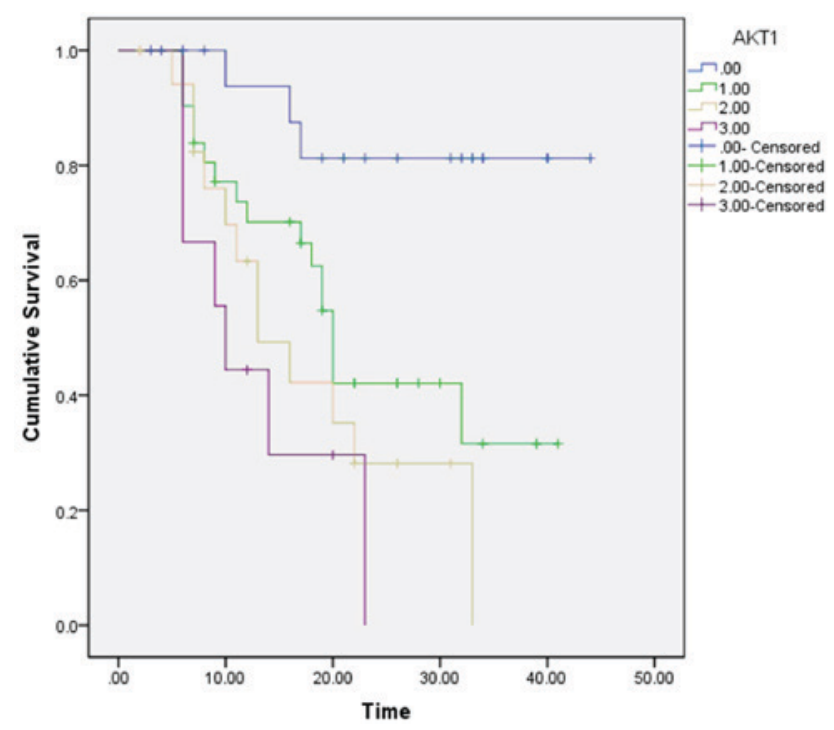

Figure 4. Survival analysis of Akt1. Blue, 0.00: Akt1- (negative), green, 1.00: Akt1+ (weakly positive), yellow, 2.00: Akt1++ (median positive) and purple 3.00: Akt1+++ (strongly positive). $\mathrm{X}$ axis, months; $\mathrm{Y}$ axis, survival rate (\%).

plot; Akt1-positive patients exhibited a relatively lower mean survival rate than Akt1-negative cases (Fig. 4).

In multivariate analysis, Enneking staging (relative risk $[R R]=3.01)$, response to chemotherapy $(R R=3.20)$ and $R A N K$ expression $(\mathrm{RR}=8.77)$ were confirmed as independent prognostic factors for osteosarcoma, as shown in Table III.

\section{Discussion}

There is evidence that the RANK/RANKL/OPG axis functions as a regulator of diverse carcinogenesis (15). The downstream molecular mechanisms, however, are not completely understood. Akt1 is a RANK downstream signaling molecule and promotes tumor progression $(16,17)$. The present study utilized immunohistochemical methods 
to investigate the clinicopathological relevance of RANK and Akt1 expression to OS progression and their correlations.

The results are partially consistent with those of previous studies concerning RANK signaling. Since RANK signaling differs between physiological conditions and tumorigenesis (17), the well-known cancer-promoting factor Akt1, which is known to be activated by RANK in vitro, was selected for investigation in the present study. Since cytological experiments have revealed that RANK activation upregulates Akt1 and thus promotes OS cell motility by interplaying with RANKL (17), it is likely that RANK expression may be linked to cancer metastasis. In addition, RANK promotes OS cell proliferation through Akt and extracellular signal-regulated kinase $1 / 2$ (17).

The correlation analysis of the present study supports the hypothesis that RANK and Akt1 may work together to promote OS progression. However, in the present study, it is difficult to determine whether RANK and Akt1 contribute together in cancer proliferation or metastasis or independently due to the limited size of the cohort. For the univariate analysis, the present study failed to find statistical significance between RANK or Akt1 expression and gender, age and the histological type of patients. From consideration of these results and those of previous consistent studies (18), it is hypothesized that RANK or Akt1 may not be tightly involved in human OS initiation but may function in tumor progression. It seems possible that the activity of the entire RANK/RANKL/OPG axis is involved in tumor progression and may be useful for predicting survival and response to chemotherapy. In addition, in vitro analyses have indicated that, through the activation of RANK, RANKL modulates gene expression involved in a wide range of cell activities such as protein synthesis and DNA regulation to affect cellular fate $(5,15)$. However, RANK signaling did not significantly affect the proliferation of OS cell lines in an in vitro model (15). By contrast, OPG gene therapy has been shown to directly inhibit OS progression through the inhibition of RANKL (7).

The long-term survival rate in OS patients treated with conventional chemotherapy has been reported as $28 \%$ (19). Clinical parameters such as tumor size and histological response to chemotherapy appear to be of prognostic value (20). Therefore, future efforts should be placed on the prediction of non-responders to conventional chemotherapy. In these patients, combining neoadjuvant chemotherapy with targeted medicine, such as chromatin-modifying drugs (21) or RANKL-related antibodies $(10,12)$ may improve the survival rate. In conclusion, the present study demonstrates that RANK expression in OS is correlated with clinicopathological characteristics and it is an independent prognostic factor in OS progression.

\section{Acknowledgements}

The present study was supported by the National Natural Science Foundation of China (no. 81371934). The authors would like to thank Dr Li Zhang in the Department of Statistics of Central South University for guidance with the statistical analysis and Dr Ling Chen in the Department of Pathology for helping with sample dissection.

\section{References}

1. Gatta G, Capocaccia R, Stiller C, Kaatsch P, Berrino F and Terenziani M; EUROCARE Working Group: Childhood cancer survival trends in Europe: A EUROCARE working group study. J Clin Oncol 23: 3742-3751, 2005.

2. Dougall WC, Glaccum M, Charrier K, Rohrbach K, Brasel K, De Smedt T, Daro E, Smith J, Tometsko ME, Maliszewski CR, et al: RANK is essential for osteoclast and lymph node development. Genes Dev 13: 2412-2424, 1999.

3. Dougall WC: RANKL signaling in bone physiology and cancer. Curr Opin Support Palliat Care 1: 317-322, 2007.

4. Lee JA, Jung JS, Kim DH, Lim JS, Kim MS, Kong CB, Song WS, Cho WH, Jeon DG, Lee SY and Koh JS: RANKL expression is related to treatment outcome of patients with localized, high-grade osteosarcoma. Pediatr Blood Cancer 56: 738-743, 2011.

5. Dougall WC and Chaisson M: The RANK/RANKL/OPG triad in cancer-induced bone diseases. Cancer Metastasis Rev 25: 541-549, 2006.

6. Feeley BT, Liu NQ, Conduah AH, Krenek L, Roth K, Dougall WC, Huard J, Dubinett S and Lieberman JR: Mixed metastatic lung cancer lesions in bone are inhibited by noggin overexpression and rank: Fc administration. J Bone Miner Res 21: 1571-1580, 2006.

7. Baud'huin M, Duplomb L, Ruiz Velasco C, Fortun Y, Heymann D and Padrines M: Key roles of the OPG-RANK-RANKL system in bone oncology. Expert Rev Anticancer Ther 7: 221-232, 2007.

8. Bago-Horvath Z, Schmid K, Roessler F, Nagy-Bojarszky K, Funovics P and Sulzbacher I: Impact of RANK signalling on survival and chemotherapy response in osteosarcoma. Pathology 46: 411-415, 2014.

9. Nicholson KM and Anderson NG: The protein kinase B/Akt signalling pathway in human malignancy. Cell Signal 14: 381-395, 2002.

10. Kostenuik PJ, Nguyen HQ, McCabe J, Warmington KS Kurahara C, Sun N, Chen C, Li L, Cattley RC, Van G, et al: Denosumab, a fully human monoclonal antibody to RANKL, inhibits bone resorption and increases BMD in knock-in mice that express chimeric (murine/human) RANKL. J Bone Miner Res 24: 182-195, 2009.

11. Linnerth-Petrik NM, Santry LA, Petrik JJ and Wootton SK: Opposing functions of Akt isoforms in lung tumor initiation and progression. PLoS One 9: e94595, 2014.

12. Paplomata E and O'Regan R: The PI3K/AKT/mTOR pathway in breast cancer: Targets, trials and biomarkers. Ther Adv Med Oncol 6: 154-166, 2014.

13. Gonzalez E and McGraw TE: The Akt kinases: Isoform specificity in metabolism and cancer. Cell Cycle 8: 2502-2508, 2009.

14. Kundu ZS: Classification, imaging, biopsy and staging of osteosarcoma. Indian J Orthop 48: 238-246, 2014.

15. Jones DH, Nakashima T, Sanchez OH, Kozieradzki I, Komarova SV, Sarosi I, Morony S, Rubin E, Sarao R, Hojilla CV, Komnenovic V, et al: Regulation of cancer cell migration and bone metastasis by RANKL. Nature 440: 692-696, 2006.

16. Liu W, Wang SQ, Wei S, Sun L and Feng X: Receptor activator of NF-kappaB (RANK) cytoplasmic motif, 369PFQEP373, plays a predominant role in osteoclast survival in part by activating Akt/PKB and its downstream effector AFX/FOXO4. J Biol Chem 280: 43064-43072, 2005.

17. Beristain AG, Narala SR, Di Grappa MA and Khokha R: Homotypic RANK signaling differentially regulates proliferation, motility and cell survival in osteosarcoma and mammary epithelial cells. J Cell Sci 125: 943-955, 2012.

18. Soedarsono N, Rabello D, Kamei H, Fuma D, Ishihara Y, Suzuki M, Noguchi T, Sakaki Y, Yamaguchi A and Kojima T: Evaluation of RANK/RANKL/OPG gene polymorphisms in aggressive periodontitis. J Periodontal Res 41: 397-404, 2006.

19. Bernthal NM, Federman N, Eilber FR, Nelson SD, Eckardt JJ, Eilber FC and Tap WD: Long-term results ( $>25$ years) of a randomized, prospective clinical trial evaluating chemotherapy in patients with high-grade, operable osteosarcoma. Cancer 118: 5888-5893, 2012.

20. Mankin HJ, Hornicek FJ, Rosenberg AE, Harmon DC and Gebhardt MC: Survival data for 648 patients with osteosarcoma treated at one institution. Clin Orthop Relat Res: 286-291, 2004.

21. Thayanithy V, Park C, Sarver AL, Kartha RV, Korpela DM, Graef AJ, Steer CJ, Modiano JF and Subramanian S: Combinatorial treatment of DNA and chromatin-modifying drugs cause cell death in human and canine osteosarcoma cell lines. PLoS One 7: e43720, 2012. 